\title{
Focal Functional and
}

\section{Microstructural Changes of}

Photoreceptors in Eyes with

Acute Zonal Occult Outer

Retinopathy

\author{
Keitetsu So ${ }^{a}$ Kei Shinoda ${ }^{a}$ Celso Soiti Matsumoto ${ }^{a}$ \\ Shingo Satofuka ${ }^{a}$ Yutaka Imamura $^{\mathrm{b}}$ Atsushi Mizota ${ }^{\mathrm{a}, \mathrm{c}}$ \\ aDepartment of Ophthalmology, Teikyo University School of Medicine, University \\ Hospital Itabashi, Tokyo, b Department of Ophthalmology, Teikyo University \\ School of Medicine, University Hospital Mizonokuchi, Kawasaki, and 'Department \\ of Ophthalmology, Juntendo University Urayasu Hospital, Chiba, Japan
}

\section{Key Words}

Acute zonal occult outer retinopathy - Perimetry - Multifocal electroretinogram - Optical coherence tomography . External limiting membrane - Photoreceptor inner and outer segment line $\cdot$ IS/OS line $\cdot$ Cone outer segment tips

\begin{abstract}
Purpose: Acute zonal occult outer retinopathy (AZOOR) is characterized by an acute zonal loss of outer retinal function with minimal ophthalmoscopic changes in one or both eyes. We present a patient with AZOOR whose ultrastructural and functional findings were followed for 8 months.

Case: A 22-year-old woman developed an acute central scotoma in her right eye. Her best-corrected visual acuity (BCVA) was $0.5 \mathrm{OD}$ and $1.2 \mathrm{OS}$. The ophthalmoscopic examinations, fluorescein angiography, and full-field electroretinograms (ERGs) were normal in both eyes. The amplitudes of the multifocal ERGs (mfERGs) were attenuated in the area corresponding to the scotoma. Spectral domain optical coherence tomography showed an absence of both the inner and outer segment (IS/OS) line of the photoreceptors and the cone outer segment tip (COST) line between the IS/OS line and the retinal pigment epithelium. These changes were seen in the area corresponding to the scotoma. One month later, the scotoma disappeared and the BCVA improved to 1.2 OD. The mfERGs increased to almost the same amplitude as the fellow eye. The IS/OS line became discernible but the COST line was still absent. The ophthalmological findings of the right macula remained normal during the 11-month follow-up period.
\end{abstract}


Conclusions: Our findings indicate that the selective loss of the IS/OS and the COST lines is probably the morphological alterations corresponding with the reduced BCVA and the mfERGs in the areas of the visual field defects in the acute phase of AZOOR. But in the recovery phase, only the abnormality of the COST line is a subclinical sign for the disease. These findings should be important in understanding and evaluating the pathological mechanism in other outer retinal diseases.

\section{Introduction}

Acute zonal occult outer retinopathy (AZOOR) is a uni- or bilateral disease characterized by an acute zonal loss of outer retinal function with minimal ophthalmoscopic changes [1]. It is associated with other clinical diseases including acute idiopathic blind spot enlargement syndrome, acute macular neuroretinopathy, multiple evanescent white dot syndrome, multifocal choroiditis, and panuveitis. These are all believed to have a similar etiology and are called the AZOOR complex [1-4].

Optical coherence tomography (OCT) of eyes with the AZOOR complex showed abnormalities in the microstructures of the outer retina, e.g., disruption or loss of the external limiting membrane (ELM), the inner and outer segment (IS/OS) line, or the cone outer segment tip (COST) line [5]. The COST line is a thin highly reflective line located between the IS/OS junction and the retinal pigment epithelium (RPE), and it is absent in the areas where the visual field defects are present [4, 6-11]. However, the pathophysiological interpretation of the intactness of this line, and the diagnostic value of the COST line have not been established.

We have examined a patient with AZOOR and followed the changes in the ultrastructure and function in the area of the focal retinal lesion.

\section{Case Report}

A 22-year-old Japanese woman complained of a central scotoma in the right eye. Her best-corrected visual acuity (BCVA) was 0.5 with a correction of -4.0 diopter in the right eye and 1.2 with -4.5 diopter in the left eye. The intraocular pressure was $14 \mathrm{~mm} \mathrm{Hg} \mathrm{OU}$. The anterior segment was normal. Ophthalmoscopy and fluorescein angiography (FA) showed that the retina and choroid were normal. Humphrey perimetry tested with central 30-2 program and SITA-Fast strategy (Humphrey Field Analyzer, Carl Zeiss, San Leandro, Calif., USA) for central 30 degree demonstrated a relative central scotoma in the right eye (fig. 1). Single flash, full-field mixed rod and cone electroretinograms (ERGs), photopic cone ERGs, and oscillatory potentials were recorded according to ISCEV standard for ERG [12] using LED built-in contact lens electrode (EW-102, Mayo, Inazawa, Japan). All responses were normal in both eyes. The multifocal ERGs (mfERGs) were recorded with a VERIS recording system (EDI, San Mateo, Calif., USA). The visual stimulus consisted of 61 hexagonal elements scaled in size to give approximately equal-amplitude local responses with eccentricity. The amplitudes of the mfERGs were reduced in the area corresponding to the visual field defects in the right eye (fig. 1).

The entire macular area was scanned with a spectral domain optical coherence tomography (SDOCT) instrument (Cirrus OCT; Carl Zeiss Meditec, Inc.) with scan lengths of $9 \mathrm{~mm}$. High quality images were obtained by using the 5 -line raster mode. The ELM was distinct in the SD-OCT images, whereas the IS/OS lines and the COST line between the IS/OS and RPE in the macula of the right eye were not detected (fig. 1). These ophthalmological findings and negative serological findings were compatible with AZOOR, and no special treatment was used. 
One month later, the central scotoma disappeared and repeated Humphrey perimetry detected no abnormality anymore. The visual acuity improved to 1.2 in the right eye. The amplitudes of the mfERGs recovered to the same level as that of the healthy fellow eye (fig. 2 ). The IS/OS line became discernible but the COST line was still absent (fig. 2). These ophthalmological findings, except the ultrastructure of the right macula, remain normal during the 11-month follow-up examinations.

\section{Discussion}

Several authors have suggested that the primary site of the pathology in the AZOOR complex is the photoreceptors $[1-4,9]$. In support of this, the time domain OCT images showed that the IS/OS line was irregular and indistinct in a patient with AZOOR, and the authors suggested that the pathology was in the photoreceptor outer segments [6].

Recent improvements in the resolution of OCT have allowed better evaluations of the intraretinal microstructures in several retinal diseases [13]. The OCT images showed that the presence of a continuous IS/OS line was due to well-restored photoreceptor cells [5], and the integrity of the ELM was correlated with the morphologic changes in the photoreceptor cell bodies and Müller cells [14, 15]. Srinivasan et al. [5] reported that the highly reflective line between the IS/OS junction and the RPE was the COST line, and it was visible because of the different lengths of the cone and rod OSs. A correlation was found between the site of the visual impairments and the loss of the foveal COST line in several retinal diseases including macular hole, occult macular dystrophy, and the AZOOR complex $[11,16,17]$. All of these reports focused on the microstructure and reported that the integrity of the microstructure was correlated with the BCVA, i.e., absence of IS/OS, ELM, and/or COSTs was present in eyes with decreased BCVA and their intactness coexisted with good BCVA. In addition, a significant correlation was present between these morphological changes and the BCVA.

Our findings showed a discrepancy between the microstructural findings and the visual functions, i.e., good BCVA, normal visual fields, and normal-size mfERGs in spite of an absence of the COST line. To the best of our knowledge, our findings are the first to compare the microstructure of the photoreceptors and visual function in the course of AZOOR.

Kondo et al. [18] reported that the attenuated mfERGs remained even after the visual acuity and visual field had only partially recovered to normal levels in a patient with acute idiopathic blind spot enlargement syndrome. They attributed the discrepancy between perimetry and the mfERGs to methodological differences, i.e., the mfERGs are elicited by suprathreshold stimulus intensities from localized regions, whereas static perimetry employs near threshold stimuli. The mfERGs sum the activity of sensitive and insensitive cells from the stimulated retina, whereas the psychophysical threshold is determined by the activity of the most sensitive cells [19].

In our case, an absence of the COST line was observed even after recovery of the cone function detected by perimetry and mfERGs. These findings suggest that the COST line is the most sensitive parameter for the pathology of the retina. Alternatively, the absence of the COST line does not necessarily mean a clinically discernible dysfunction of the central cone photoreceptors once the disease develops. What the absence of the COST line means and whether it will recover in this case are unknown. Further longitudinal studies on a 
large number of patients are required to determine the underlying pathology and the meaning of the integrity of the photoreceptor microstructure detected by SD-OCT.

Our findings were made on a single case of AZOOR, and they cannot be extended to all cases of AZOOR until further studies are done. But observations of the integrity of outer retinal microstructure including the ELM, IS/OS line, and the COST line in correlation with functional parameters would be informative and clinically relevant to the understanding of the pathologic mechanism and to the determination of the severity or stage of different retinal diseases.

\section{Acknowledgement}

Support of this study was provided by Research Grants on Sensory and Communicative Disorders from the Ministry of Health, Labor, and Welfare, Japan.

\section{Disclosure Statement}

No author has a financial or proprietary interest in any material or method mentioned. 


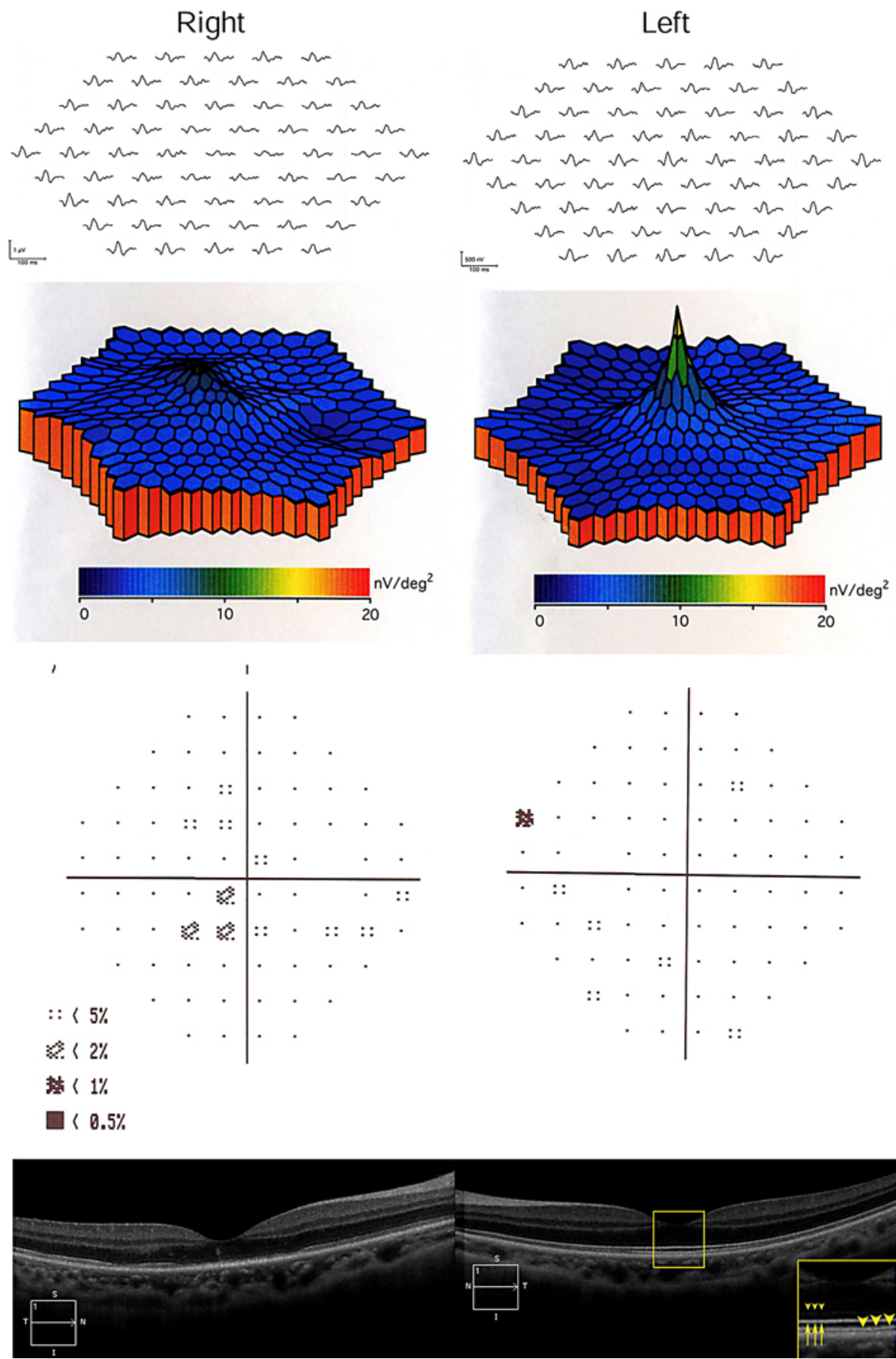

Fig. 1. Clinical findings of a patient at the acute phase of AZOOR. The visual acuity was 0.5 OD and 1.2 OS. First and second rows: mfERGs of the 61 response arrays and 3-dimensional plot of the mfERGs, respectively. These mfERGs show the reduced responses in the area corresponding with the visual field defect. The third row shows the pattern deviation probability map of the Humphrey static visual field tested with central 30-2 program and SITA-Fast strategy (Humphrey Field Analyzer, Carl Zeiss, San Leandro, Calif., USA) for central 30 degree. The mean deviation was $-1.04 \mathrm{~dB}$ in the right eye and $-2.25 \mathrm{~dB}$ in the left eye, and the probability map shows a reduced sensitivity in the central area of the right eye. The bottom row shows the Fourierdomain optical coherence tomographic images from the affected right eye and normal left eye. These images show that both the photoreceptor IS/OS line and the COST line between the IS/OS line and RPE are absent in the macula area of the right eye. The IS/OS line, COST line, and the RPE/Bruch membrane are intact in the left eye. Arrowheads indicate ELM. Arrows indicate IS/OS. Large arrowheads indicate COST line. 

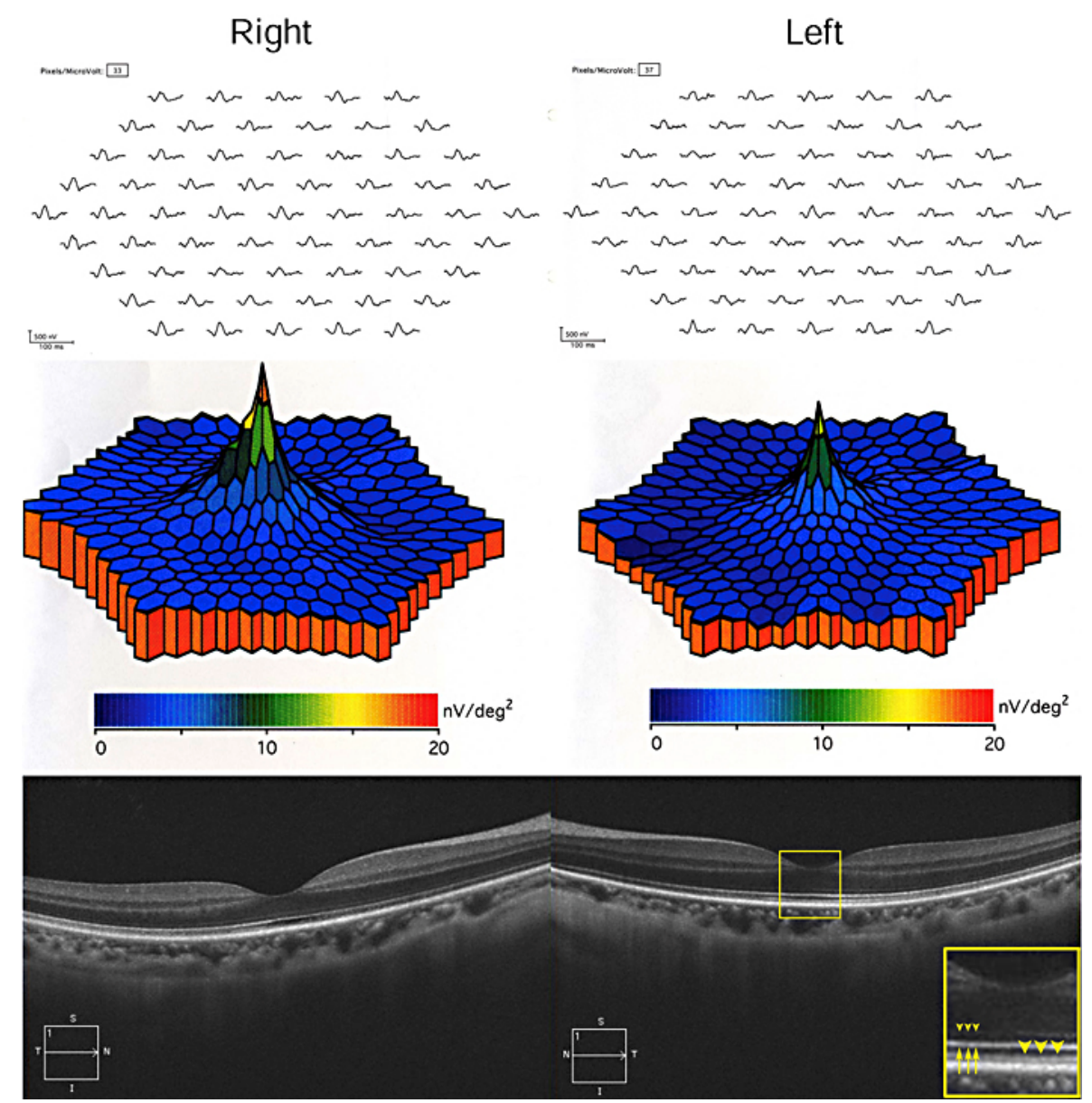

Fig. 2. Clinical findings of our AZOOR patient at the recovery phase. Visual acuity was $1.2 \mathrm{OU}$. The top and the second row panels are 61 response arrays and 3-dimensional plot of the multifocal electroretinograms, respectively. These show a recovery of the functions in the central area of the right eye. The bottom panels are Fourier-domain optical coherence tomographic images from both eyes, showing that the border of the photoreceptor IS/OS line is clearly discernible but the COST line is still absent in the foveal area of the right eye. The IS/OS line, COST line, and the RPE/Bruch membrane are intact in the left eye. Arrowheads indicate ELM. Arrows indicate IS/OS. Large arrowheads indicate COST line.

\section{References}

1 Gass JD: Acute zonal occult outer retinopathy. Donders Lecture: The Netherlands Ophthalmological Society, Maastricht, Holland, June 19, 1992. J Clin Neuroophthalmol 1993;13:79-97.

2 Gass JD, Agarwal A, Scott IU: Acute zonal occult outer retinopathy: a long-term follow-up study. Am J Ophthalmol 2002;134:329-339.

-3 Gass JD: Are acute zonal occult outer retinopathy and the white spot syndromes (AZOOR complex) specific autoimmune diseases? Am J Ophthalmol 2003;135:380-381.

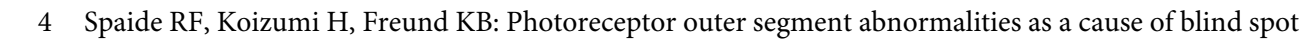
enlargement in acute zonal occult outer retinopathy-complex diseases. Am J Ophthalmol 2008;146:111-120.

-5 Srinivasan VJ, Monson BK, Wojtkowski M, et al: Characterization of outer retinal morphology with highspeed, ultrahigh-resolution optical coherence tomography. Invest Ophthalmol Vis Sci 2008;49:1571-1579.

6 Li D, Kishi S: Loss of photoreceptor outer segment in acute zonal occult outer retinopathy. Arch Ophthalmol 2007;125:1194-1200. 
-7 Takai Y, Ishiko S, Kagokawa H, Fukui K, Takahashi A, Yoshida A: Morphological study of acute zonal occult outer retinopathy (AZOOR) by multiplanar optical coherence tomography. Acta Ophthalmol 2009;87:408418.

-8 Zibrandtsen N, Munch IC, Klemp K, Jørgensen TM, Sander B, Larsen M: Photoreceptor atrophy in acute zonal occult outer retinopathy. Acta Ophthalmol 2008;86:913-916.

-9 Fujiwara T, Imamura Y, Giovinazzo VJ, Spaide RF: Fundus autofluorescence and optical coherence tomographic findings in acute zonal occult outer retinopathy. Retina 2010;30:1206-1216.

10 Ohta K, Sato A, Fukui E: Spectral domain optical coherence tomographic findings at convalescent stage of acute zonal occult outer retinopathy. Clin Ophthalmol 2009;3:423-428.

-11 Sugahara M, Shinoda K, Matsumoto CS, Satofuka S, Hanazono G, Imamura Y, Mizota A: Outer retinal microstructure in case of acute idiopathic blind spot enlargement syndrome. Case Rep Ophthalmol 2011;2:116-122.

12 Marmor MF, Fulton AB, Holder GE, Miyake Y, Brigell M, Bach M: Standard for clinical electroretinography (2008 update). Doc Ophthalmol 2009;118:69-77.

13 Byeon SH, Kang SY: Interpretation of outer retina appearance in high-resolution optical coherence tomography. Am J Ophthalmol 2009;147:185-186.

14 Lim JI, Tan O, Fawzi AA, Hopkins JJ, Gil-Flamer JH, Huang D: A pilot study of Fourier-domain optical coherence tomography of retinal dystrophy patients. Am J Ophthalmol 2008;146:417-426.

15 Wakabayashi T, Oshima Y, Fujimoto H, Murakami Y, Sakaguchi H, Kusaka S, Tano Y: Foveal microstructure and visual acuity after retinal detachment repair. Ophthalmology 2009;116:519-528.

-16 Wakabayashi T, Fujiwara M, Sakaguchi H, Kusaka S, Oshima Y: Foveal microstructure and visual acuity in surgically closed macular holes: spectral-domain optical coherence tomographic analysis. Ophthalmology 2010;117:1815-1824.

17 Park SJ, Woo SJ, Park KH, Hwang JM, Chung H: Morphologic photoreceptor abnormality in occult macular dystrophy on spectral-domain optical coherence tomography. Invest Ophthalmol Vis Sci 2010;51:3673-3679.

18 Kondo N, Kondo M, Miyake Y: Acute idiopathic blind spot enlargement syndrome: prolonged retinal dysfunction revealed by multifocal electroretinogram technique. Am J Ophthalmol 2001;132:126-128.

19 Hood DC, Zhang X: Multifocal ERG and VEP responses and visual fields: comparing disease-related changes. Doc Ophthalmol 2000;100:115-137. 